\title{
Correlation between gliotoxin production and virulence of Aspergillus fumigatus in Galleria mellonella
}

\author{
Emer P. Reeves ${ }^{1}$, C.G.M. Messina ${ }^{2}$, S. Doyle ${ }^{1} \&$ K. Kavanagh ${ }^{1}$ \\ ${ }^{1}$ Department of Biology, National Institute for Cellular Biotechnology, National University of Ireland Maynooth, \\ County Kildare, Ireland; ${ }^{2}$ Laboratory of Haematology and Oncology, Instituto Superiore di Sanita, Viale Regina \\ Elena 299, 00161 Roma, Italy
}

Received 13 June 2003; accepted in final form 27 Octobber 2003

\begin{abstract}
Aspergillus fumigatus is a pathogenic fungus capable of causing both allergic lung disease and invasive aspergillosis, a serious, life-threatening condition in neutropenic patients. Aspergilli express an array of mycotoxins and enzymes which may facilitate fungal colonisation of host tissue. In this study we investigated the possibility of using the insect, Galleria mellonella, for in vivo pathogenicity testing of Aspergillus species. Four clinical isolates of Aspergillus fumigatus and a single strain of Aspergillus niger were characterised for catalase and elastase activity and for the production of gliotoxin. Gliotoxin is an immunosuppressive agent previously implicated in assisting tissue penetration. Results illustrated a strain dependent difference in elastase activity but no significant difference in catalase activity. Gliotoxin production was detected in vitro and in vivo by Reversed Phase-High Performance Liquid Chromatography, with highest amounts being produced by A. fumigatus ATCC 26933 (350 ng/mg hyphae). Survival probability plots (Kaplan-Meier) of experimental groups infected with Aspergillus conidia indicate that G. mellonella is more susceptible to fungal infection by A. fumigatus ATCC 26933, implicating a critical role for gliotoxin production rather than growth rate or enzymatic activity in the virulence of $A$. fumigatus in this model.
\end{abstract}

Key words: gliotoxin, Aspergillus fumigatus, Galleria mellonella, fungal infection, immunosuppression

Abbreviations: Aspergillus fumigatus, A. fumigatus; Galleria mellonella, G. mellonella; Minimal Essential Medium Eagle, MEM; High Performance Liquid Chromatography, HPLC; Malt Extract Agar, MEA.

\section{Introduction}

Fungal infections are of increasing importance in severely neutropenic and immunosuppressed patients because of their high incidence and the associated high mortality rate. Despite the development of triazole anti-fungals and amphotericin B formulations the mortality associated with fungal infection remains high at $60-90 \%$. Aspergillosis is one such opportunistic infection, which can occur in immunosuppressed patients and is most commonly caused by the fungus Aspergillus fumigatus [1].

A. fumigatus, as well as some Candida [2] and Penicillium [3] species, produce gliotoxin a member of the epipolythiodioxopiperazine class of fungal metabolites [4]. The activity of gliotoxin is attributable to a reactive disulfide bond component in the molecule, which can undergo redox cycling, resulting in the production of oxygen radicals. A broad spectrum of biological activity has been reported for gliotoxin which include the prevention of macrophage adherence, followed by the induction of apoptosis [5, 6]. Inhibition of phagocytosis by rodent macrophages and mitotic stimulation of lymphocytes has been shown, an effect unrelated to induction of apoptosis [7] but which may assist fungal colonization. Other activities associated with gliotoxin include inhibition of fungal and bacterial growth [8] and viral replication [9]. From a clinical perspective, gliotoxin is important since it has been reported as being produced in tissues of mice and turkey poults [10] following development of invasive aspergillosis. 
Models of invasive aspergillosis were first developed in experimental murine systems [11], with evidence suggesting that the innate mammalian immune response is involved in the defence against fungal pathogens. Key features involved in innate immunity are found to be homologous in insects and vertebrates $[12,13]$. The insect cellular processes such as phagocytosis [14], production of reactive oxygen species [15] and anti-microbial peptides, bear strong similarity to those produced by phagocytes of mammals, giving evidence of an ancient immune system found in the ancestor of all bilaterian animals. Most immunological data for insects have been reported from Drosophila melanogaster [16] however in the Greater Wax moth Galleria mellonella, various immunorelevant protein molecules have been described and this system is being increasingly used as a model for assessing the virulence of a range of microorganisms [17, 18]. For example, larvae of G. mellonella have been successfully used to evaluate the pathogenicity of lipopolysaccharide-deficient mutants of Pseudomonas aeruginosa [19] and a good correlation exists between the virulence of $P$. aeruginosa in Galleria larvae and in mice. Larvae of G. mellonella have also been used to assess the virulence of Candida albicans and strong agreement has been established between the results obtained in insects and mice [20].

This paper describes the use of G. mellonella larvae as a screening mechanism for testing the virulence of A. fumigatus. Studies confirm the production of gliotoxin by four clinical isolates of A. fumigatus and a single isolate of $A$. niger and demonstrate that $G$. mellonella is more susceptible to fungal infection by strains of Aspergillus producing elevated levels of gliotoxin.

\section{Material and methods}

\section{Fungal strains and culture conditions}

Clinical isolates of Aspergillus fumigatus used in this study included ATCC 26933, 16424, 13073 and 14109 (obtained from the American Type Culture Collection, Maryland, USA), one strain of Aspergillus niger (environmental isolate) and the yeast Saccharomyces cerevisiae YJM 128 (A gift from Dr. K. Clemons, San Jose, USA) [20]. Aspergillus spores were grown in Minimal Essential Medium Eagle (Sigma Aldrich Chemical Co., Dublin, Ireland) supplemented with 5\% (v/v) Foetal Calf Serum (Sigma Aldrich) at $37{ }^{\circ} \mathrm{C}$ and $200 \mathrm{rpm}$, for up to 4 days. Stocks were maintained on Malt Extract Agar (MEA) (Oxoid Ltd.). $S$. cerevisiae was grown to stationary phase (approximately $\left.1 \times 10^{8} / \mathrm{ml}\right)$ in YEPD broth $(2 \%(\mathrm{w} / \mathrm{v})$ glucose, $2 \%(\mathrm{w} / \mathrm{v})$ bactopeptone (Difco), $1 \%(\mathrm{w} / \mathrm{v})$ yeast extract (Oxoid) at $30{ }^{\circ} \mathrm{C}$ and $200 \mathrm{rpm}$. Stocks were maintained on YEPD agar (2\% (w/v) Agar, Difco).

\section{Growth curve for Aspergillus}

MEA plates containing sporulating Aspergillus cultures were washed with $10 \mathrm{ml}$ of $0.1 \%(\mathrm{v} / \mathrm{v})$ Tween 80 (Merck) in Phosphate Buffered Saline (PBS, pH 7.2) (Sigma Aldrich) to isolate conidia. Conidia were washed twice in sterile PBS, centrifuged (1,500 g, 5 min in a Beckman GS- 6 centrifuge) and counted using an haemocytometer. Flasks containing MEM $(100 \mathrm{ml})$ were inoculated with $1 \times 10^{5}$ Aspergillus conidia and incubated at $37^{\circ} \mathrm{C}$ and $200 \mathrm{rpm}$. A flask was removed every $24 \mathrm{~h}$ and the contents filtered through a Whatman No. 1 filter in a Buchner funnel and air-dried. A growth curve was constructed of dry fungal biomass versus incubation time.

\section{Characterisation of Aspergillus strains}

Catalase activity of the five Aspergillus strains was determined using Aspergillus niger catalase (Sigma Aldrich) as a standard, the difference in absorbance $\left(\Delta A_{240}\right)$ per unit time being a measure of the catalase activity [21]. Elastase activity was quantified using the elastase-specific substrate $N$-succinylalanyl-alany-prolyl-leucine $p$-nitroanilide (Sigma Aldrich) [22]. Substrate (final concentration, $2.5 \mathrm{mM}$ ) was added to fungal hyphae (final concentration, 0.33 $\mathrm{mg} / \mathrm{ml}$ protein) in buffer (100 mM Tris- $\mathrm{HCl}, 200 \mathrm{mM}$ $\mathrm{NaCl}, 0.05 \%$ (v/v) Triton X-100, pH 7.8) in a 96-well, flat bottomed microtiter plate at $37^{\circ} \mathrm{C}$. The $\mathrm{A}_{405}$ was read every $60 \mathrm{~s}$ for $10 \mathrm{~min}$.

\section{Preparation of extracts for detection of gliotoxin by Reversed Phase-HPLC}

Hyphae of Aspergillus were removed from the MEM culture medium by filtration and an equal volume of chloroform (Hyper Solv, BDH) added to the filtrates $(10 \mathrm{ml})$. Following continual mixing for $30 \mathrm{~min}$, the chloroform fraction was collected and evaporated to dryness. For detection of gliotoxin, dried extracts were dissolved in $200 \mu \mathrm{l}$ methanol (Hyper Solv, BDH) and stored at $-70{ }^{\circ} \mathrm{C}$ until assayed. Gliotoxin was detected by Reversed Phase-HPLC (Spectra-Physics). 
The mobile phase was $34.9 \%(\mathrm{v} / \mathrm{v})$ acetonitrile (Hyper Solv, BDH), $0.1 \%$ (v/v) trifluoroacetic acid (Sigma Aldrich) and 65\% (v/v) deionized-distilled water. The gliotoxin extract $(20 \mu \mathrm{l})$ was injected onto a $\mathrm{C} 18 \mathrm{Hew}-$ lett Packard column. A standard curve of peak area versus gliotoxin concentration was constructed using gliotoxin standards (50, 100 and $200 \mathrm{ng} / \mathrm{ml}$ dissolved in methanol, (Sigma Aldrich). The amount of gliotoxin in the samples was calculated from the standard curve.

\section{Inoculation of insects with conidia of Aspergillus}

Sixth instar larvae of G. mellonella were obtained from the Meal Worm Company (Sheffield, England). Larvae were stored in wood shavings in the dark at $15^{\circ} \mathrm{C}$ prior to use. All larvae were used within 3 weeks of receipt. Larvae between 0.2 and $0.4 \mathrm{~g}$ in weight were employed in all assays. Larvae were injected with $1 \times 10^{4}$ conidia in $20 \mu 1$ PBS into the haemocoel through the last left pro-leg as described previously using a Myjector U-100 Insulin needle (Terumo) [17]. Larvae were placed in a sterile petri dish and incubated in the dark at $30{ }^{\circ} \mathrm{C}$. Mortality rates were determined over a 5 day period. Larval death was assessed by the absence of movement and discolouration of the cuticle $[17,20]$. The extraction of gliotoxin from tissue samples infected with Aspergillus was as described by Richard et al. [10]. In brief, 100 infected larvae were homogenized in $5 \mathrm{ml}$ of $\mathrm{H}_{2} \mathrm{O}$. The homogenizer was rinsed twice with $10 \mathrm{ml}$ of $6 \mathrm{~N} \mathrm{HCL}$ and pooled to give $25 \mathrm{ml}$. Chloroform $(200 \mathrm{ml})$ was added and the mixture stirred at room temperature for $30 \mathrm{~min}$. The sample was poured into a separatory funnel and the gliotoxin extracted in the lower chloroform layer. Detection and quantification of gliotoxin was as described above.

\section{Statistical analysis}

Survival of larvae after injection was analysed by Kaplan-Meier survival analysis. Catalase and elastase activities were analysed by Student's two tailed $t$-test.

\section{Results}

Growth curves for the five Aspergillus isolates (Figure 1) showed the exponential growth phase began at $12 \mathrm{~h}$ and extended until $48 \mathrm{~h}$. The stationary phase

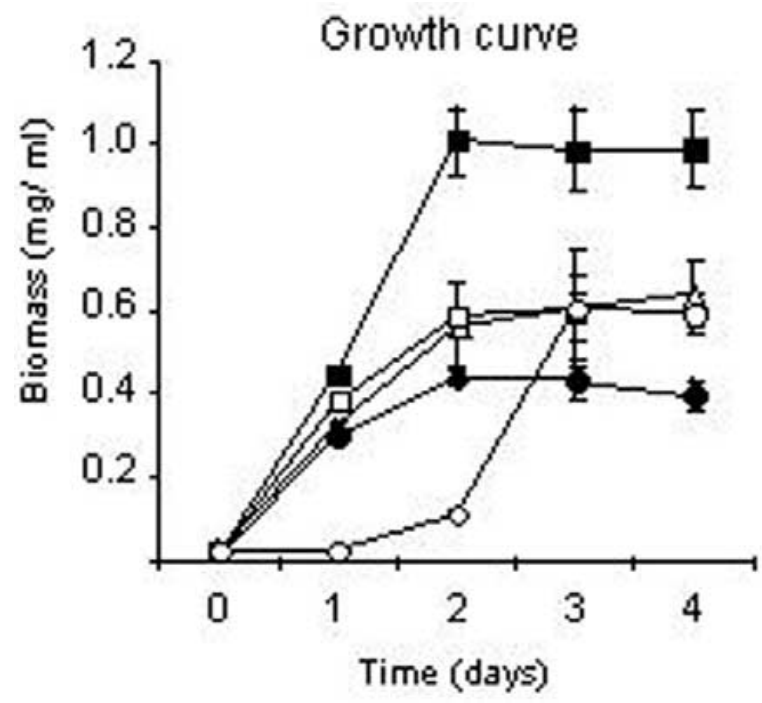

Figure 1. Growth curves. Growth curve of Aspergillus isolates in $\mathrm{MEM}$ at $37^{\circ} \mathrm{C}, \boldsymbol{O}=\mathrm{ATCC} 26933, \bigcirc=$ A. niger, $\mathbf{\square}=16424, \triangle=$ $13073, \square=14109$.

was reached at approximately $72 \mathrm{~h}$ with a greater biomass being obtained for A. fumigatus ATCC 16424 $(1.0 \mathrm{mg} / \mathrm{ml}$ of culture).

Characterization of the different Apergillus strains showed them to vary in enzymatic activity. Quantification of elastase activity demonstrated that $A$. niger and A. fumigatus ATCC 14109 exhibited elevated levels of activity (25-30 U/mg hyphae), whereas isolates ATCC 26933, 16424 and 13073 produced between 10 and 20 $\mathrm{U}$ of elastase activity per mg of fungal hyphae (Figure 2a). Between the different Aspergillus isolates no significant difference was found in the level of catalase activity, with a standard level of approximately 0.25 $\mathrm{U} / \mathrm{min} / \mathrm{mg}$ hyphae activity calculated (Figure $2 \mathrm{~b}$ ).

Production of gliotoxin by A. fumigatus has previously been detected at temperatures ranging from $30^{\circ} \mathrm{C}$ [23] to $37^{\circ} \mathrm{C}$ [24]. The conventional method of Reversed Phase-HPLC quantification was used for the determination of gliotoxin production [25]. All isolates of Aspergillus were found to produce gliotoxin with production starting after $24 \mathrm{~h}$ (Figure 3 ). Strain ATCC 26933 produced more gliotoxin than the other 4 strains of Aspergillus (350 ng/mg hyphae, $P<$ 0.001 ), with maximum gliotoxin production occurring at approximately $72 \mathrm{~h}$ incubation. A subsequent decline in levels was observed which may have been due to catabolism or instability of the compound.

An investigation into the pathogenicity of the five strains of Aspergillus was performed using the G. mellonella virulence model. Figure 4a shows the mor- 

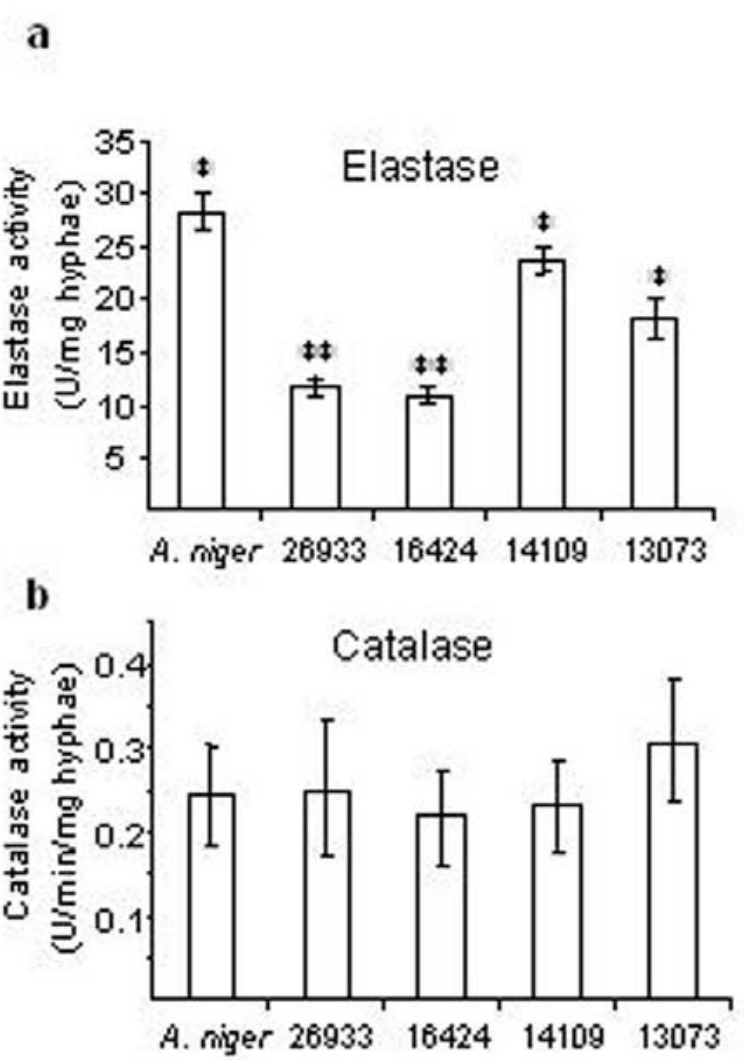

Figure 2. Enzymatic activity. (a) Elastase activity of A. niger and four strains of A. fumigatus. $* *=$ no significant difference between isolates ATCC 26933 and $16424(P=0.1), *=$ significant difference between A. niger, ATCC 14109 and 13073 relative to 26933 and $16424(P<0.01)$. (b) Catalase activity of the 5 isolates of Aspergillus $(P=0.09)$. All results are means \pm s.e.m. from three separate measurements.

tality of Galleria following injection with Aspergillus conidia. The control, a non-virulent strain of $S$. cerevisiae [17] and A. fumigatus strains ATCC 13073 and 16424 had minimal effect on larval mortality. This was in marked contrast to the A. fumigatus strain ATCC 14109 and A. niger which killed approximately $20 \%$ of the infected larvae within $24 \mathrm{~h}$ after infection with $1 \times 10^{4}$ conidia.

The results reveal elevated mortality rates for larvae inoculated with the clinical isolate A. fumigatus ATCC 26933. Furthermore, within $48 \mathrm{~h}$ all larvae inoculated with this strain had been killed. This strain was found to produce the highest levels of gliotoxin (Figure 4b), indicating a likely correlation between gliotoxin production and virulence in G. mellonella.

In order to establish a more direct link between gliotoxin production and virulence, further studies were made on the occurrence of gliotoxin in larvae of $G$.

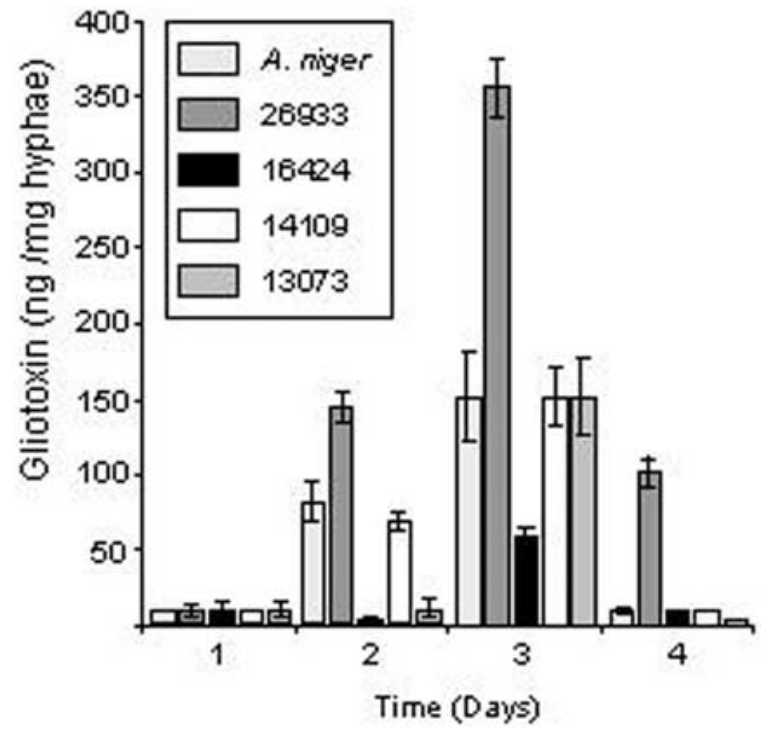

Figure 3. Quantification of gliotoxin production. Gliotoxin production as a function of fungal hyphal biomass. All results are means \pm s.e.m. from three measurements. A statistically significant difference was found for ATCC 26933 compared to all other strains at days 2,3 and $4(P<0.001)$.

mellonella infected with the fungus. Results showed that the toxin was formed during growth of A. fumigatus, as gliotoxin was found in larvae infected with the gliotoxin-producing strains and was not found in tissues from healthy uninfected larvae. As depicted in Figure $4 \mathrm{~b}$ various levels of gliotoxin was found in $G$. mellonella tissue samples following inoculation with $1 \times 10^{4}$ conidia of the different Aspergillus isolates. The levels of gliotoxin ranged from $250-2400 \mathrm{ng} / \mathrm{g}$ of insect mass; two Aspergillus isolates, ATCC 16424 and ATCC 13073 produced no detectable levels. The highest concentration of gliotoxin detected followed inoculation with A. fumigatus ATCC 26933 (2400 $\mathrm{ng} / \mathrm{g}$ ) at a time corresponding to $50 \%$ larval death (Figure 4a). Infection with $1 \times 10^{4} \mathrm{~A}$. fumigatus ATCC 14109 or A. niger resulted in similar (250$280 \mathrm{ng} / \mathrm{g}$ ) gliotoxin concentrations. Collectively these results indicate a likely correlation between gliotoxin production and virulence of A. fumigatus ATCC 26933 in G. mellonella.

\section{Discussion}

The fact that there are many airborne fungi, not implicated in disease suggests that $A$. fumigatus may produce specific virulence factors that are important in helping the pathogenic fungus to colonize tissue. Several lines 

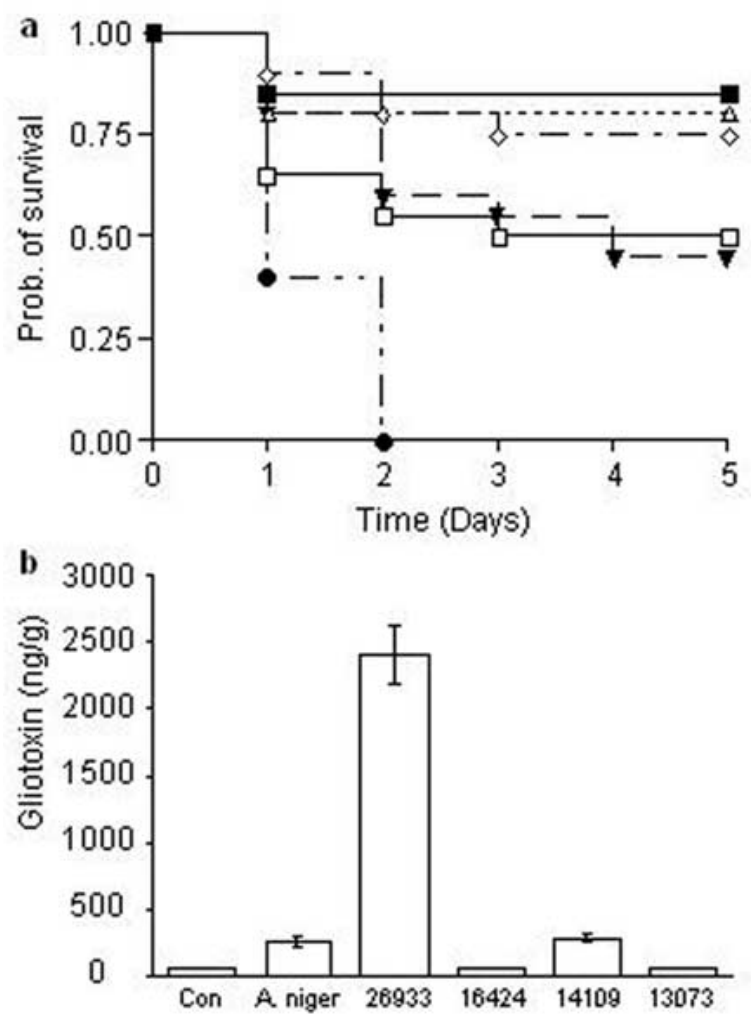

Figure 4. Susceptibility of G. mellonella larvae to fungal infection. (a) Survival probability plots (Kaplan-Meier) of larvae injected with $1 \times 104$ conidia of the Aspergillus isolates $(n=20)$. The rates of decreased probability of survival of larvae injected with A. fumigatus ATCC 26933 was significantly different from all other isolates $(P<0.05) . \quad=$ ATCC 26933, $\square=$ A. niger, $\boldsymbol{\nabla}=14109$, $\diamond=16424, \Delta=13073, \mathbf{\square}=S$. cerevisiae. (b) In vivo production of gliotoxin as a function of larval biomass. Larvae of G. mellonella were injected with $1 \times 10^{4}$ conidia of $A$. fumigatus ATCC 26933, 16424, 14109, 13073, and A. niger and gliotoxin production compared to uninfected larvae (Con).

of evidence suggest a role for gliotoxin in the pathogenesis of aspergillosis, as this metabolite is produced by over $95 \%$ of pathogenic isolates of $A$. fumigatus.

Neutrophils and alveolar macrophages are the main cellular components of the vertebrate immune system responsible for the defence against $C$. albicans [26] and A. fumigatus [27]. They eliminate pathogens by phagocytosis, the release of reactive oxygen intermediates and activation of proteolytic enzymes [26, 28]. Similar cellular processes have been described for phagocytes of insects, with receptors on the surface of plasmatocytes and granulocytes exhibiting striking similarity to receptors on mammalian phagocytes [14]. A. fumigatus can escape local host defences by synthesizing metabolites that inhibit these immune defences. Müllbacher and Eichner described the inhibition of phagocytosis by gliotoxin [6], while others have demonstrated that gliotoxin causes morphological alterations to macrophages [29]. The wax moth G. mellonella has previously been used to test the virulence of a variety of microorganisms $[17,18$, $20]$ and in this study we employed $G$. mellonella to differentiate between pathogenic Aspergillus species and to determine if the production of an immunosuppressive agent, such as gliotoxin, could further compromise the infected host. On comparing the lethality of different strains, it should be noted that the growth rate of each strain might have the potential to affect pathogenesis. It was observed that the Aspergillus isolate ATCC 16424 which possessed the fastest growth rate, exhibited a virulence level equivalent to that of the non-virulent isolate of S. cerevisiae [20].

Catalase is an antioxidant metalloenzyme, which is almost ubiquitous among aerobic organisms and protects cells against oxidative damage caused by hydrogen peroxide. It has been reported that oxidative mechanisms are important in the killing of A. fumigatus by polymorphonuclear cells and that this fungus is sensitive in vitro to oxygen metabolites [30]. The catalase activities of Aspergillus isolates employed in this study were quantified. As all isolates possessed similar activity, an anti-virulent role for catalase in protection against oxidative killing by reactive oxygen intermediates produced by haemocytes cannot be assessed using the particular Aspergillus strains of this study.

It has been speculated that extracellular proteinases play a role in virulence, by allowing tissue invasion by fungal cells and also in resistance to phagocytic killing by the host [31]. A number of isolates of A. fumigatus obtained from a hospital environment produced extracellular elastolytic activity and immunogold localization of the elastolytic enzyme showed that $A$. fumigatus germinating and penetrating into the lungs of neutropenic mice secreted the elastolytic protease [32]. Elastase activity of the five Aspergillus isolates used in this study was quantified and although various levels of activity were observed, those strains illustrating increased elastase activity, demonstrated low levels of virulence as measured in G. mellonella.

Gliotoxin production by $A$. fumigatus has been estimated to be in the range of 20 to $80 \mu \mathrm{g} / \mathrm{ml}$ culture after approximately 4-7 days [6] and this concentration has been shown to inhibit the growth of several Gram-negative bacteria and fungi, and viral replication in vitro [33]. In this study A. fumigatus ATCC 26933 produced the highest concentration of gliotoxin 
(350 ng/mg of hyphae) after three days growth at $37^{\circ} \mathrm{C}$. Interestingly a lower concentration of gliotoxin (20-50 ng/ml) has been shown to have an inhibitory effect on phagocytosis of carbon particles by peritoneal exudates [7]. An additional observation was the detection of maximum gliotoxin production after three days of growth in vitro with maximum larval death occurring two days post infection. This temporal discrepancy may indicate that the high concentration of gliotoxin detected in vitro may not be required in vivo to obtain the level of killing observed.

The results indicate the existence of a hierarchy among the Aspergillus isolates studied here with respect to their ability to kill larvae of $G$. mellonella, i.e. ATCC $26933>$ ATCC $14109=$ A. niger $>$ ATCC $16424=$ ATCC 13073. Consistently, Aspergillus species killed larvae to a greater extent than the non-pathogenic $S$. cerevisiae and the differences in relative pathogenicity between the Aspergillus isolates, in particular A. fumigatus ATCC 26933, may be explained by the increased levels of gliotoxin produced by this isolate.

The correlation between gliotoxin production and virulence in G. mellonella was further strengthened by the observation of the occurrence of gliotoxin in larvae infected with the fungus. Recoveries of gliotoxin have been made from bovine samples and from lung tissue of turkeys (10) inoculated via the posterior thoracic air sac with A. fumigatus conidia, with levels of gliotoxin ranging from $183-6849 \mathrm{ng} / \mathrm{g}$, a concentration similar to what was found in the Galleria tissue in this study. Preliminary spiking studies indicated, approximately $40 \%$ of the gliotoxin was recovered from normal larval tissue samples to which purified gliotoxin was added at known concentrations, and therefore the relevant adjustment in calculations were made. Relative to the other Aspergillus strains, A. fumigatus ATCC 26933 produced higher concentrations of gliotoxin in vivo (Figure 3 ) than in vitro (Figure 4b), which may by due to the ability of this strain to produce increased levels of the immunosuppressive toxin when challenged by cells of the host immune response. In addition, the growth environment within the larvae may facilitate elevated gliotoxin production compared to that in culture.

Inoculation of Galleria with similar concentrations of purified gliotoxin did not appear to induce the same pathogenic effect as the growing fungus (result not shown). It is possible therefore, in cases of aspergillosis that the toxin produced by the fungus is concentrated in the tissue adjacent to the colony and assists escape from local host defences via its reported immunosuppressive role [6, 29, 34, 35].

In conclusion, the work presented here demonstrates differences in the virulence of clinical isolates of Aspergillus, as detected in larvae of G. mellonella. While the insect and mammalian immune systems differ, they share a high degree of similarity in terms of their innate immune responses [12] which is an important line of defence against fungal infection [36]. The response of insects to infection shows strong similarities to that found in mammals $[17,18]$. The information generated by the use of low gliotoxin producing strains of Aspergillus, e.g. the reduced mortality of larvae infected with ATCC 13073 compared to the high mortality of larvae infected with the high gliotoxin-producing strain ATCC 26933, suggests that gliotoxin production is a significant contributor to the pathogenicity of $A$. fumigatus in this model. We have not excluded the presence of other factors that may explain the difference in virulence between the Aspergillus isolates however with the data obtained, gliotoxin is a likely candidate, further strengthening its role in the pathogenesis of A. fumigatus-induced diseases.

\section{Acknowledgements}

This work is supported by funding from the HEA under PRTLI cycle III.

\section{References}

1. Daly P, Kavanagh K. Pulmonary aspergillosis: clinical presentation, diagnosis and therapy. B Biomed Sci 2001; 58: 197-205.

2. Saha DT, Larsen B. Clinical isolates of yeast produce a gliotoxin like substance. Mycopathologia 1991; 116: 203-208.

3. Richard J, DeBey M, Chermette R, Pier A, Hasegawa A, Lund A, Bratberg A, Padhye A, Connole M. Advances in veterinary mycology. J Med Vet Mycol 1994; 32: 169-187.

4. Waring P, Beaver J. Gliotoxin and Related Epipolythiodioxopiperazine. Gen Pharmac 1996; 27: 1311-1316.

5. Braithwaite AW, Eichner RD, Waring P, Müllbacher A. The immunomodulating agent gliotoxin causes genomic DNA fragmentation. Mol Immunol 1987; 24: 47-55.

6. Müllbacher A, Eichner RD. Immunosuppression in vitro by a metabolite of a human pathogenic fungi. Proc Natl Acad Sci USA 1984; 81: 3835-3837.

7. Waring P, Eichner RD, Müllbacher A, Sjaarda A. Gliotoxin induces apoptosis in macrophages unrelated to its antiphagocytic properties. J Biol Chem 1988; 263: 18493-18499.

8. Boutibonnes P, Auffray Y, Malherbe C, Kogbo W, Marais C. Proprietes antibacteriennes et genotoxiques de 33 mycotoxines. Mycopathologia 1984; 87: 43-49. 
9. Larin NM, Copping MP, Herbst-Laier RH, Roberts B, Wenham RMM. Antiviral activity of gliotoxin. Chemotheraphy 1965; 10: 14-25.

10. Richard JL, Dvorak TJ, Ross PF. Natural occurrence of gliotoxin in turkeys infected with Aspergillus fumigatus, Fresenius. Mycopathologia 1996; 134: 167-170.

11. Mondon P, De Champs C, Donadille A, Ambroise-Thomas $\mathrm{P}$, Grillot R. Variation in virulence of Aspergillus fumigatus strains in a murine model of invasive pulmonary aspergillosis. J Med Microbiol 1995; 45: 186-191.

12. Kang D, Liu G, Lundstrom A, Gelius E, Steiner H. A peptigoglycan recognition protein in innate immunity conserved from insects to humans. Proc Natl Acad Sci USA 1998; 95: 10078-10082.

13. Yoshimura A, Lien E, Ingalls RR, Tuomanen E, Dziarski R, Golenbock D. Cutting edge: recognition of Gram-positive bacterial cell wall components by the innate immune system occurs via Tol-like receptor 2. J Immunol 1999; 163: 1-5.

14. Vilmos P, Kurucz E. Insect Immunity: evolutionary roots of the mammalian innate immune system. Immunol Letts 1998; 62: 59-66.

15. Slepneva IA, Glupov VV, Sergeeva SV, Khramtsov VV. EPR detection of reactive oxygen species in hemolymph of Galleria mellonella and Dendrolimus superans sibiricus (Lepidoptera) larvae. Biochem Biophys Res Commun 1999; 264: 212-215.

16. Irving P, Troxler L, Heuer TS, Belvin M, Kopczynski C, Reichhart JM, Hoffmann JA, Hetru C. A genome-wide analysis of immune responses in Drosophila. Proc Natl Acad Sci USA 2001; 98: 15119-15124.

17. Cotter G, Doyle S, Kavanagh K. Development of an insect model for the in vivo pathogenicity testing of yeast. FEMS Immunol Med Microbiol 2000; 27: 163-169.

18. Jander G, Rahme LG, Ausubel FM. Positive correlation between virulence of Pseudomonas aeruginosa mutants in mice and insects. J Bacteriol 2000; 182: 3843-3845.

19. Dunphy G, Morton D, Kropinski A, Chadwick J. Patogenicity of lipopolysaccharide mutants of Pseudomonas aeruginosa for larvae of Galleria mellonella: bacterial properties associated with virulence. J Invert Pathol 1986; 47: 48-55.

20. Brennan M, Thomas DY, Whiteway M, Kavanagh K. Correlation between virulence of Candida albicans mutants in mice and Galleria mellonella larvae. FEMS Immunol Med Microbiol 2002; 341: 153-157.

21. Kettle AJ, Winterbourn CC. A kinetic analysis of the catalase activity of myeloperoxidase. Biochemistry 2001; 40: 1020410212.

22. Borger P, Koeter GH, Timmerman JAB, Vellenga E, Tomee JFC, Kauffman HF. Proteases from Aspergillus fumigatus. J Infect Dis 1999; 180: 1267-1274.

23. Nieminen SM, Maki-Paakkanen J, Hirvonen M-R, Roponen M, von Wright A. Genotoxicity of gliotoxin, a secondary metabolite of Aspergillus fumigatus, in a battery of short-term systems. Mut Res 2002; 520: 161-170.
24. Sutton P, Waring P, Müllbacher A. Exacerbation of invasive aspergillosis by the immunosuppressive fungal metabolite, gliotoxin. Immunol Cell Biol 1996; 74: 318-322.

25. Richard JL, Lyon R1, Fichtner RE, Ross PF. Use of thin layer chromatography for dection and high performance liquid chromatography for quantitating gliotoxin from rice cultures of Aspergillus fumigatus. Mycopathologia 1989; 107: 145-151.

26. Reeves EP, Hui L, Lortat Jacobs H, Messina CGM, Bolsover S, Gabella G, Potma EO, Warley A, Roes J, Segal AW. Killing activity of neutrophils is mediated through activation of proteases by $\mathrm{K}^{+}$flux. Nature 2002; 416: 291-297.

27. Washburn RG, Gallin JI, Bennett JE. Oxidative killing of Aspergillus fumigatus proceeds by parallel myeloperoxidasedependent and -independent pathways. Infect Immun 1987; 55: 2088-2092.

28. Odeberg H, Olsson I. Mechanisms for the microbicidal activity of cationic proteins of human granulocytes. Infect Immun 1976; 14: 1269-1275.

29. Eichner RD, Al Salami M, Wood PR, Müllbacher A. The effect of gliotoxin upon macrophage function. Int J Immunopharmacol 1986; 8: 789-797.

30. Leitz SM, Diamond RD. Mechanisms of resistance of Aspergillus fumigatus conidia to killing by neutrophils in vitro. $\mathrm{J}$ Infect Dis 1985; 152: 33-42.

31. Shimizu K, Kondoh Y, Tanaka K. Proteinase production and pathogenicity of Candida albicans. Microbiol Immunol 1987; 31: 1045-1060.

32. Kolattukudy PE, Lee JD, Rogers LM, Zimmerman P, Ceselski S, Fox B, Stein B, Copelan EA. Evidence for possible involvement of an elastolytic serine protease in aspergillosis. Infect Immun 1993; 61: 2357-2368.

33. Taylor A. The toxicology of sporodesim and other epipolydioxopiperasine. In: Kadis S, Ciegler A, Ajl SJ, eds. Microbiol.Toxins. Vol. 7. New York: Academic Press, 1971: 370-376.

34. Bertout S, Badoc C, Mallie M, Giaimis J, Bastide J-M. Spore diffusate isolated from some strains of Aspergillus fumigatus inhibits phagocytosis by murine alveolar macrophages. FEMS Immunol Med Microbiol 2002; 33: 101-106.

35. Sutton $\mathrm{P}$, Newcombe NR, Waring $\mathrm{P}$, Müllbacher A. In vivo immunosuppressive activity of gliotoxin, a metabolite produced by human pathogenic Fungi. Infect Immun 1994; 62: 1192-1198.

36. Aratani Y, Koyama H, Nyui S-I, Suzuki K, Kura F, Maeda N. Severe impairment in early host defence against Candida albicans in mice deficient in myeloperoxidase. Infect Immun 1999; 67: 1828-1836.

Address for correspondence: E.P. Reeves, Department of Biology, National Institute for Cellular Biotechnology, National University of Ireland Maynooth, County Kildare, Ireland

Phone: +353-1-628 5222 ext. 3137; Fax: +353-1-708 3845; E-mail: emer.reeves@may.ie 
